\title{
ANALYSIS OF THE PERMEABILITY CHARACTERISTICS ALONG ROUGH-WALLED FRACTURES USING A HOMOGENIZATION METHOD
}

\author{
BYUNG-GON CHAE ${ }^{1}$, JUNG-HAE CHOI ${ }^{1}$, YASUAKI ICHIKAWA ${ }^{2}$, and YONG-SEOK SEO ${ }^{3 *}$ \\ ${ }^{1}$ Geologic Environment Division, Korea Institute of Geoscience and Mineral Resources \\ Daejeon 305-350, Korea \\ ${ }^{2}$ Department of Environmental Design Engineering, Okayama University Okayama \\ 700-8530, Japan \\ ${ }^{3}$ Department of Earth and Environmental Sciences, Chungbuk National University \\ Cheongju 361-763, Korea \\ "Corresponding author. E-mail : ysseo@cbu.ac.kr
}

Received June 17, 2011

Accepted for Publication August 23, 2011

To compute a permeability coefficient along a rough fracture that takes into account the fracture geometry, this study performed detailed measurements of fracture roughness using a confocal laser scanning microscope, a quantitative analysis of roughness using a spectral analysis, and a homogenization analysis to calculate the permeability coefficient on the microand macro-scale. The homogenization analysis is a type of perturbation theory that characterizes the behavior of microscopically inhomogeneous material with a periodic boundary condition in the microstructure. Therefore, it is possible to analyze accurate permeability characteristics that are represented by the local effect of the facture geometry. The Cpermeability coefficients that are calculated using the homogenization analysis for each rough fracture model exhibit an irregular distribution and do not follow the relationship of the cubic law. This distribution suggests that the permeability characteristics strongly depend on the geometric conditions of the fractures, such as the roughness and the aperture variation. The homogenization analysis may allow us to produce more accurate results than are possible with the preexisting equations for calculating permeability.

KEYWORDS : Permeability Coefficient, Rough Fracture, Confocal Laser Scanning Microscope, Homogenization Analysis, Micro- and Macro-scale

\section{INTRODUCTION}

A large body of research that takes into consideration the fracture geometry, such as the orientation, roughness, aperture and connectivity, has contributed to the ability to characterize the fluid flow in a fractured rock mass. Initially, a parallel plate model with an impermeable matrix was used [1-5]. This model is based on a cubic law that implies that the flow rate is proportional to the cube of the fracture aperture. A model composed of a rough fracture's surfaces with an impermeable matrix has also been suggested [3,6-14]. This model takes into consideration the partial contact areas along the fracture walls that may result from roughness of the fracture. In addition, several studies have used an equivalent porous medium model [15-17]. This model was developed as a solution to the difficulties that are associated with accurately considering the fracture's geometry. A dual porous medium model that originated from the equivalent porous medium was also proposed for the analysis of the permeability characteristics along a fractured medium [18]. Recently, a realistic fracture model that uses the real geometry of a fracture and that simulates the permeability characteristics has been suggested [1,7,19-24].

The results of the previous studies that were mentioned above have shown that the permeability characteristics change irregularly because of the fracture geometry. Particularly in a rough rock fracture, the fluid flow occurs as a selective flow, such as a channel flow that is dependent on the fracture roughness. Therefore, a numerical analysis of the permeability characteristics should include the fracture geometry when possible.

To acquire the geometric data of a fracture, many researchers have used a profile gauge [25-34]. The roughness pattern is usually represented using a fractal dimension. In some cases, an epoxy consisting of a resin and a fluorescent material is injected into the fracture to observe the fracture roughness. Recently, several studies 
have measured roughness using graphic techniques [3537]. These studies used an electronic microscope or a video and digitized the roughness features to obtain the roughness data. However, these methods are limited in their measurement resolution or accuracy. This limitation may cause the exclusion of small-scale roughness that falls below the measurement resolution or the inaccurate digitizing of the roughness pattern because of an operator bias. These errors may lead to invalid results because of measurement inaccuracies.

The purpose of the present study was to calculate the permeability of a single fracture while taking the true fracture geometry into consideration. The fracture roughness was measured using a confocal laser scanning microscope (CLSM). The CLSM can collect roughness data very accurately with a high resolution. The roughness data that were measured using the CLSM were quantitatively analyzed using the Fourier spectral analysis. These data were used to reconstruct a fracture model for numerical analysis using a homogenization analysis (HA) method. The HA method is a perturbation theory that was developed to characterize the behavior of a microscopically inhomogeneous material that involves periodic microstructures [38,39]. The HA permeability coefficient was calculated based on the local fracture geometry and the local material properties (the water viscosity). The HA method considers flow characteristics in both micro scale and macro scale by introducing the incompressible Navier-Stokes equation. The method calculates characteristic velocity and characteristic pressure in a unit cell using a micro scale equation, and then calculates the HA-permeability coefficient. It draws a macro scale equation, namely the HA-flow equation, by using the averaged material velocity and the HA-permeability coefficient.

\section{DETAILED ANALYSIS OF THE FRACTURE ROUGHNESS}

\subsection{Fracture Roughness}

The specimens in the present study were composed of Jurassic coarse-grained granite. These specimens were collected from cores drilled in the Iksan area in the midwestern part of Korea. The six core specimens, which contained a single, fresh fracture that was parallel or subparallel to the long axis of the core, were studied in detail. They were referred to as GRA, GRB, GRC, GRD, GRE, and GRF and were $5.5 \times 11.0 \mathrm{~cm}$ in size (Figure 1).

The fracture roughness was measured using a confocal laser scanning microscope (CLSM; Olympus OLS 1100) to collect high resolution digital data on the surface roughness (See Figure 1 in [40]). The CLSM has a high level of resolution and contrast in the direction of the light axis because of its confocal optics. This microscope offers 2-D and 3-D images and a variety of other features, including improvements in the image quality, line width and shape measurements.

The wavelength of the OLS 1100 that was used in the present study was very short, as short as $488 \mathrm{~nm}$. The scanning method of this microscope is a light polarization method that uses two galvano meter scanner mirrors. The resonant galvano mirrors in this microscope enable highspeed, high-resolution imaging of a wide area. The wide field of view of this microscope makes it easy to locate the desired observation position. The non-confocal observation option produces images with large focal depths without focal effects, whereas the confocal observation option produces clear images with small focal depths [40].

In the present study, the sampling spacing was $2.5 \mu \mathrm{m}$ for the $\mathrm{x}$ - and $\mathrm{y}$-directions. The highest level of resolution of measurement for the $\mathrm{z}$ direction was $0.05 \mu \mathrm{m}$, which is a higher resolution than those of the pre-existing methods. To collect the 2-D roughness data, one scan line was placed on the fracture's surface along the long axis of the fracture. The resolutions for the $\mathrm{x}$ - and $\mathrm{y}$-directions were fixed at $1,024 \times 768$ pixels $(2.56 \times 1.92 \mathrm{~mm}$ in area). In the present study, the resolution for the z-direction was $10 \mu \mathrm{m}$ because the difference in the roughness from the highest peak to the lowest peak was large. Figure 2 shows an example of a fracture roughness that was measured with a CLSM that contained noise.

\subsection{Roughness Analysis using the Fourier Spectral Analysis}

Fourier spectral analysis was conducted to quantitatively determine the roughness characteristics. The Fourier transform distills complicated components into simple frequency components and represents the magnitudes of the analyzed frequencies. Therefore, it is possible to identify

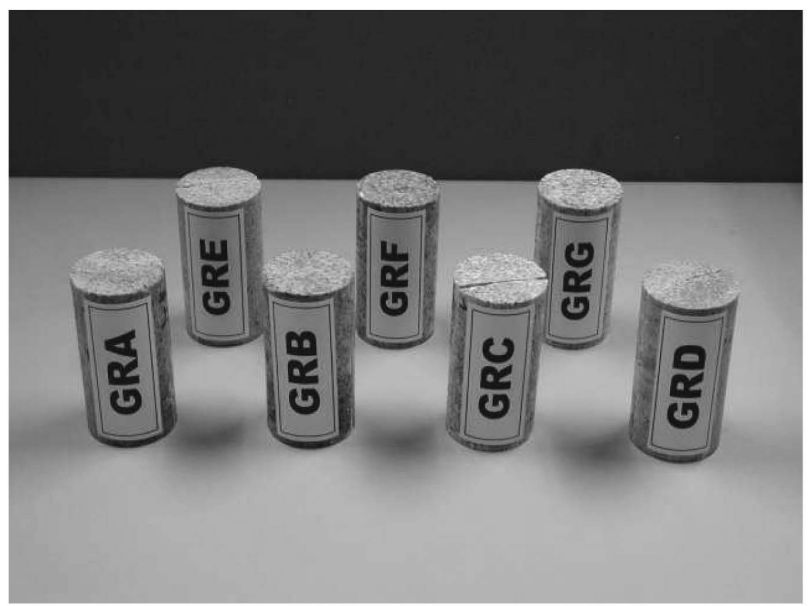

Fig. 1. Core Specimens used for the Measurements of Roughness 
the composition of the frequency and to determine the most effective frequency in the roughness data [40].

On the basis of the roughness data, the power spectra were acquired using the fast Fourier transform (Figure 3(a)). The power spectra $\left|G\left(k_{n}\right)\right|$ of the original data has an exponential distribution, with high amplitudes in the low frequency domain that gradually decrease in amplitude as they approach the high frequency domain. The components of the long wavelengths contribute to the determination of the overall features of the fracture roughness, whereas the high frequency components affect the small scale roughness.

A noise reduction procedure is necessary to obtain smoothed spectra from the roughness data (Figure 3(b)).
The present study performed noise reduction using Parzen's lag window. The Parzen window applies a weight to the central part of the moving window in the frequency domain. Therefore, this window has a smoothed peak at its center and very narrow side lobes at the margins of the moving window $[40,41]$.

After the Fourier spectral analysis and the noise reduction procedures were performed for all of the data for each specimen, a reconstruction procedure of the roughness geometry was performed using the influential frequencies of all of the components of the frequency. There was an abrupt point of change in the spectrum pattern that was due to the remnant noise in the filtered spectrum (Figure 3(c)). After the selection of the changing threshold

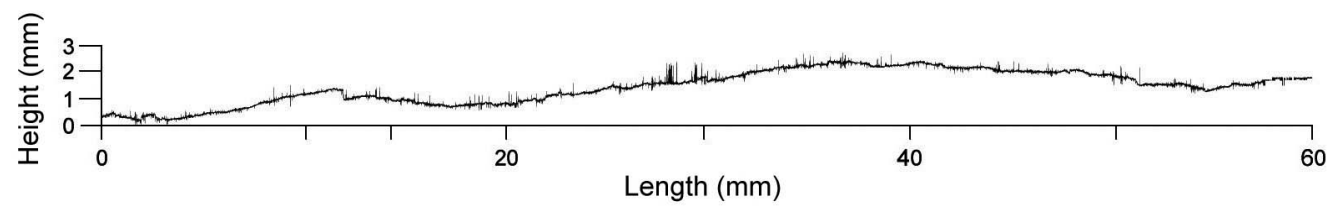

Fig. 2. An Example of the Roughness Measured using the CLSM (Left Part of GRD)

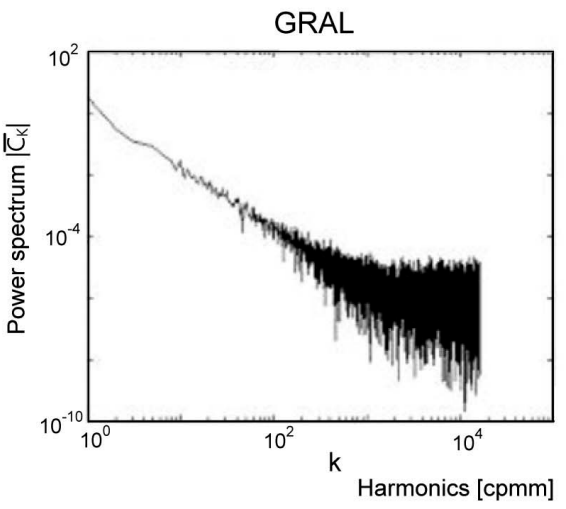

a

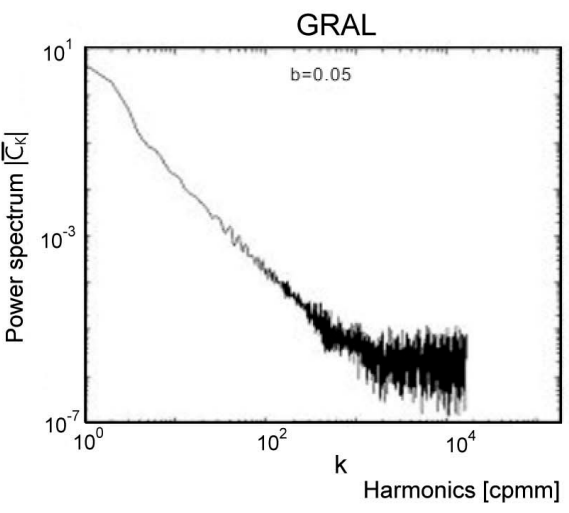

b

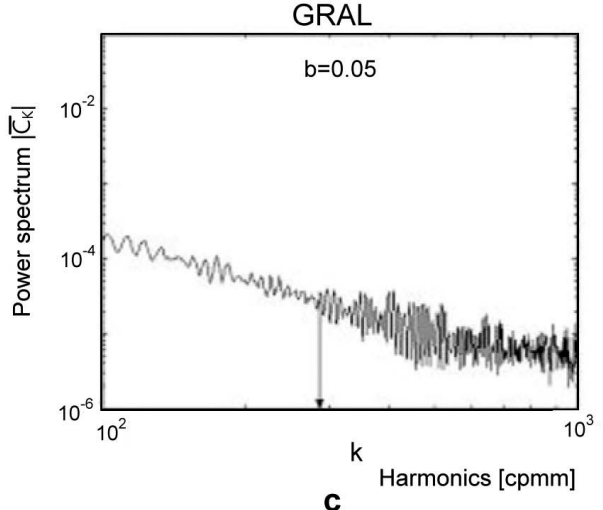

Fig. 3. Results of the Fast Fourier Transform for the Left Part of GRA (GRAL), (a) Noise-containing Spectrum, (b) Noise-reduced Spectrum, and (c) Threshold of the Low Pass Filter 
for the spectrum pattern, an overlapping cosine curve for each frequency was drawn in the lower frequency area in relation to the threshold. The overlapping cosine curve represents a reconstructed smooth roughness pattern that is without noise (Figure 4). The reconstructed roughness was in agreement with the measured roughness data. The reconstructed roughness features were used to develop the fracture models for the analyses of the fluid flow along a fracture.

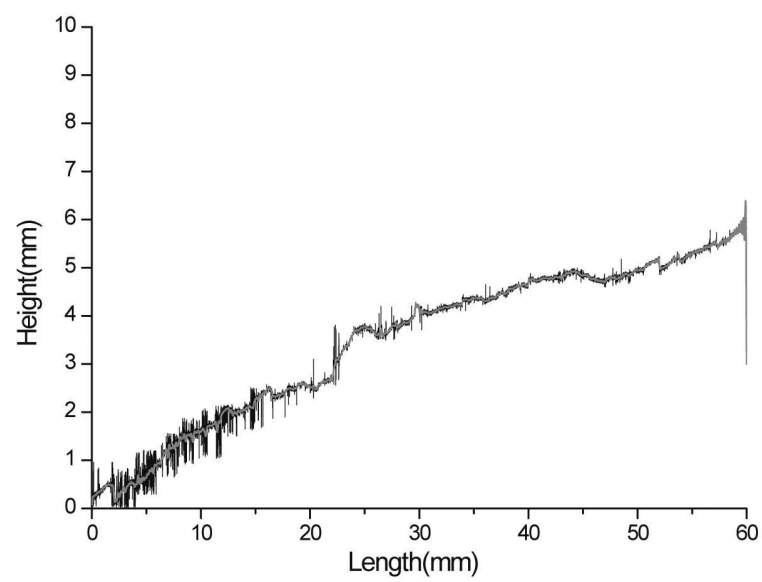

Fig. 4. Comparison of the Measured Roughness Data of GRAL, which Contained Noise (Black) in Addition to the Smoothed Roughness Data that were Filtered using a Low Pass Filter (Red)

\section{THE HOMOGENIZATION ANALYSIS METHOD}

For the calculation of the permeability coefficients with the consideration of the detailed fracture geometry, the present study introduced the homogenization analysis (HA) method. The HA method was applied to the flow problem with periodic micro-structures [38,39] (Figure 5). For this problem, the Navier-Stokes equation was assumed for the local flow field.

Let us introduce the local coordinate system $y$, which is related to the global system, $\boldsymbol{x}$, by $\boldsymbol{y}=\boldsymbol{x} / \varepsilon$. Here, $\varepsilon$ is a micro parameter. The following incompressible NavierStokes flow field is introduced:

$$
\begin{gathered}
-\frac{\partial P^{\varepsilon}}{\partial x_{i}}+\eta \frac{\partial^{2} V_{i}^{\varepsilon}}{\partial x_{k} \partial x_{k}}+F_{i}=0 \text { in } \Omega_{\varepsilon f}, \\
\frac{\partial V_{i}^{\varepsilon}}{\partial x_{i}}=0 \quad \text { in } \Omega_{\varepsilon f} .
\end{gathered}
$$

where $V_{i}^{\varepsilon}$ is the velocity vector with the shearing viscosity $\eta, P$ is the pressure, $F_{i}$ is the body force vector, and $\Omega_{\varepsilon f}$ is the water flow region in the global coordinate system.

An asymptotic expansion is introduced as follows:

$$
\begin{aligned}
& V_{i}^{\varepsilon}(\boldsymbol{x})=\varepsilon^{2} V_{i}^{0}(\boldsymbol{x}, \boldsymbol{y})+\varepsilon^{3} V_{i}^{1}(\boldsymbol{x}, \boldsymbol{y})+\ldots, \\
& P^{\varepsilon}(\boldsymbol{x})=P^{0}(\boldsymbol{x}, \boldsymbol{y})+\varepsilon P^{1}(\boldsymbol{x}, \boldsymbol{y})+\ldots,
\end{aligned}
$$

where $V_{i}^{\alpha}(\boldsymbol{x}, \boldsymbol{y})$ and $P^{i}(\boldsymbol{x}, \boldsymbol{y})(\alpha=0,1, \ldots)$ are $\mathbf{Y}$-periodic functions, such as $V_{i}^{\alpha}(\boldsymbol{x}, \boldsymbol{y})=V_{i}^{\alpha}(\boldsymbol{x}, \boldsymbol{y}+\mathbf{Y})$ and $P^{i}(\boldsymbol{x}, \boldsymbol{y})=$

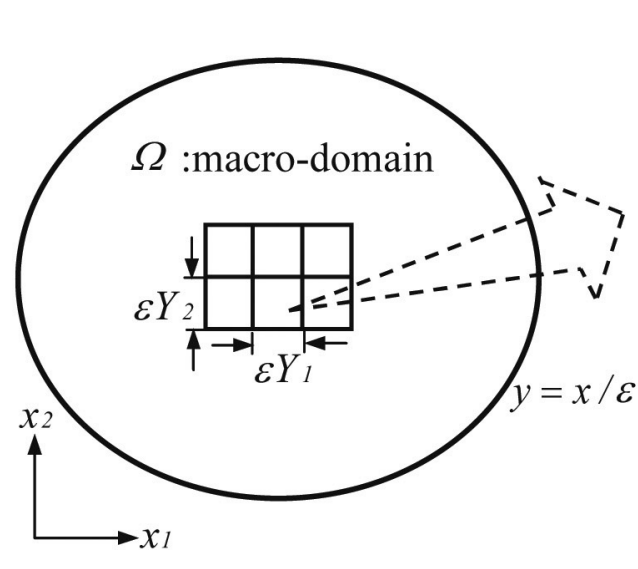

Macro-scale problem with

periodic micro-structure

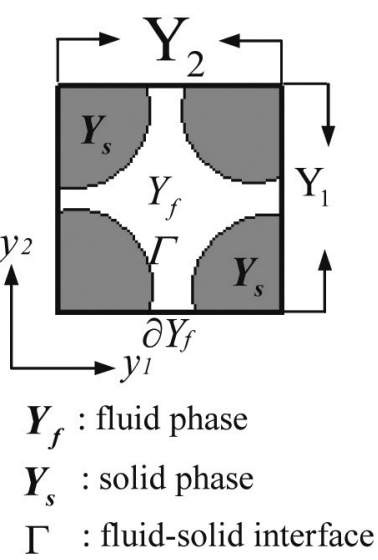

$\partial Y_{f}$ : periodic B.C.

Fig. 5. Macro- and micro-scale concepts in the HA 
$P^{i}(\boldsymbol{x}, \boldsymbol{y}+\mathbf{Y})$, with the size of a unit cell Y. Because $\boldsymbol{y}=\boldsymbol{x} / \varepsilon$, the differentiation changes to

$$
\frac{\partial}{\partial x_{i}} \Rightarrow \frac{\partial}{\partial x_{i}}+\frac{1}{\varepsilon} \frac{\partial}{\partial y_{i}}
$$

Substituting Equations (3) and (4) into Equation (1) and taking $\varepsilon \rightarrow 0$ yields the following:

$\varepsilon^{-1}$-term: $\frac{\partial P_{0}}{\partial y_{i}}=0 \Rightarrow P^{0}(\boldsymbol{x}, \boldsymbol{y})=P^{0}(\boldsymbol{x})$ in $Y_{f}$

and

$$
\varepsilon^{0} \text {-term: }-\frac{\partial P^{1}}{\partial y_{i}}+\eta \frac{\partial^{2} V_{i}^{0}}{\partial y_{k} \partial y_{k}}=\frac{\partial P^{0}}{\partial x_{i}}-F_{\mathrm{i}} \text { in } Y_{f}
$$

Similarly, Equation (3) gives

$$
\varepsilon^{1} \text {-term: } \frac{\partial V_{i}^{0}}{\partial y_{i}}=0 \text { in } Y_{f} \text { and }
$$

and

$$
\varepsilon^{2} \text {-term: } \frac{\partial V_{i}^{0}}{\partial x_{i}}+\frac{\partial V_{i}^{1}}{\partial y_{j}}=0 \text { in } Y_{f}
$$

Next, a separation of these variables is introduced for Equation (6) by

$$
\begin{aligned}
& V_{i}^{k}=\left(F_{k}(\boldsymbol{x})-\frac{\partial P^{0}(\boldsymbol{x})}{\partial x_{k}}\right) v_{i}^{k}(\boldsymbol{y}), \\
& P^{1}=\left(F_{k}(\boldsymbol{x})-\frac{\partial P^{0}(\boldsymbol{x})}{\partial x_{k}}\right) p^{k}(\boldsymbol{y}),
\end{aligned}
$$

where $v_{i}^{k}(\boldsymbol{y})$ and $p^{k}(\boldsymbol{y})(k=1,2,3)$ are referred to as the characteristic velocity and the characteristic pressure, respectively. Then, Equation (6) is changed into a PDE of only $\boldsymbol{y}$ :

$$
-\frac{\partial p^{k}}{\partial y_{i}}+\eta \frac{\partial^{2} v_{i}^{k}}{\partial y_{j} \partial y_{j}}+\delta_{i k}=0 \text { in } Y_{f}
$$

In a similar manner, the mass conservation law (7) is written as

$$
\frac{\partial v_{i}^{k}}{\partial y_{i}}=0 \quad \text { in } \quad Y_{f}
$$

Equations (10) and (11) are referred to as the 'micro scale equations' (MiSE) for the water flow problem.

Now, an averaging operation is introduced for Equation (10), and the following, Darcy's Law, is obtained using the HA:

$$
\tilde{V}_{i}^{0}=K_{j i}\left(F_{j}-\frac{\partial P^{0}}{\partial x_{j}}\right), \quad K_{j i}=\tilde{v}_{i}^{j}=\frac{1}{|\boldsymbol{Y}|} \int_{Y_{f}} v_{i}^{j} d y,
$$

where $\tilde{V}_{i}^{o}$ is the averaged velocity in the unit cell $(|\boldsymbol{Y}|$, volume of unit cell), and $K_{j i}$ is referred to as the HA-permeability. It can be shown that $K_{j i}$ is symmetrical and non-negatively definite. The same averaging is applied to Equation (8), and the second term disappears because of the periodic condition of $V_{i}^{1}$. Therefore, the following 'macroscale equation' (MaSE), called the HA-flow equation, is specified:

$$
\frac{\partial \tilde{V}_{i}^{0}}{\partial x_{i}}=0 \text { or } \frac{\partial}{\partial x_{i}}\left[K_{j i}\left(F_{j}-\frac{\partial P^{0}}{\partial x_{j}}\right)\right]=0 \text { in } \Omega .
$$

The true pressure $P$ and velocity $V_{i}$ are calculated, in terms of a first order approximation, by Equation (3) as

$$
V_{i}^{\varepsilon}(\boldsymbol{x})=\varepsilon^{2} V_{i}^{0}(\boldsymbol{x}, \boldsymbol{y}), \quad P^{\varepsilon}(\boldsymbol{x}) \cong P^{0}(\boldsymbol{x}) .
$$

In the geotechnical engineering field, the following empirical Darcy's Law is commonly used:

$$
\tilde{V}_{i}^{\prime}=-K_{i j}^{\prime} \frac{\partial H}{\partial x_{j}} ; \quad H=\frac{P}{\rho g}+\zeta,
$$

where $\tilde{V}_{i}^{\prime}$ is the average velocity in the classical sense (referred to as the seepage velocity), $H$ is the total head, $P$ is the pore pressure, $\zeta$ is the elevation head, $g$ is the gravitational acceleration and $\rho$ is the mass density of the water, which is assumed to be constant because of its incompressibility. Comparing this with Equations (13) to (15), the correspondence

$$
\tilde{V}_{i}^{\prime}=\tilde{V}_{i}^{\varepsilon} \cong \varepsilon^{2} \tilde{V}_{i}^{0},
$$

is found. Thus, the following relationship between the HA-permeability $K_{j i}$ and the C-permeability $K_{j i}^{\prime}$ is specified:

$$
K_{i j}^{\prime}=\varepsilon^{2} \rho g K_{i j} .
$$

Note that this C-permeability coefficient $K_{j i}{ }_{j i}$ can be compared with the conventional, experimental and theoretical values. The validity of the HA-permeability concept has been illustrated in previous works $[38,42]$.

\section{COMPUTATION OF THE PERMEABILITY COEFFICIENTS OF THE ROUGH FRACTURE MODELS USING A HOMOGENIZATION ANALYSIS}

The C-permeability coefficients were computed using several rough fracture models. The important objective of the homogenization analysis was to understand the changes of the permeability characteristics that are dependent on the roughness patterns. The fracture models were constructed with a consideration of the fracture roughness that was analyzed using the fast Fourier transform.

The HA permeability characteristics were determined for various roughness conditions. For several types of roughness features that were measured using the CLSM, the upper fracture wall was displaced at intervals of $1 \mathrm{~mm}$ in the shearing direction. This shear displacement was 
introduced for five stages, which resulted in various aperture values along the fracture. The permeability coefficient was calculated at every stage of this displacement. The fracture models of each specimen are shown in Figure 6. These models represent various roughness features and apertures because of the displacement. Every model exhibits different geometrical features at each stage of the displacement.

The mean aperture values at each stage of the displacement for all of the specimens are shown in Table 1. The majority of the values indicate a positive proportional relationship between the shear displacement and the mean aperture value. However, the case of specimen GRC is different. In this sample, the mean aperture value decreased as the shearing displacement increased. This relationship is due to the inclination direction of the fracture of GRC.
In other words, the fracture is inclined from left to right, but the shearing is applied from right to left.

The results of the $\mathrm{C}$-permeability coefficients at each stage of the shear displacement are shown in Table 2. It was determined that the permeability coefficients were irregularly ranged from $10^{-4}$ to $10^{-1} \mathrm{~cm} / \mathrm{sec}$, whereas the coefficients of the previous parallel plate models were uniformly distributed within a certain range. This difference of permeability between this study and the parallel plate models was due to complicated changes in the roughness and the aperture values, which increased the shear displacement in the current models. The relationships between the square of the mean aperture, $b^{2}$, and the calculated permeability are shown in Figure 7 . The nonlinear relationship in Figure 7 indicates that the cubic law was not suitable for the rough fracture case [43].

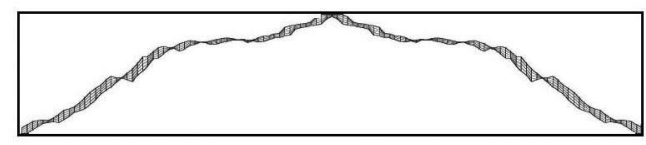

a

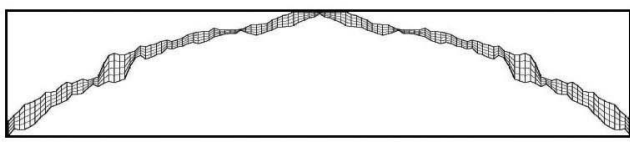

C

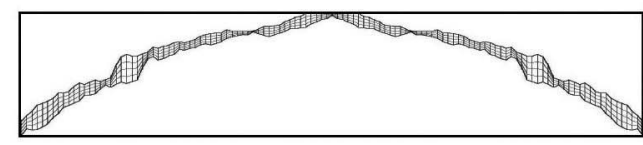

b

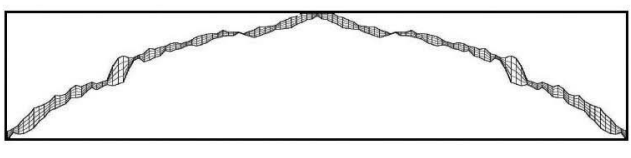

d

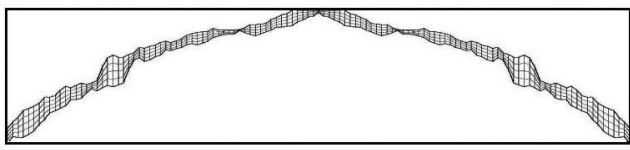

e

Fig. 6. Fracture Models of GRA Showing Various Roughness Features and Apertures at Each Stage. Exaggerated 100 Times in the Vertical Direction. (a) 1st Stage, (b) 2nd Stage, (c) 3rd Stage, (d) 4th Stage, and (e) 5th Stage

Table 1. Mean Aperture Values of the Five Stages of Shear Displacement.

\begin{tabular}{c|c|c|c|c|c|r}
\hline \multirow{2}{*}{$\begin{array}{c}\text { Shear disp. } \\
(\mathrm{mm})\end{array}$} & \multicolumn{9}{|c}{ Mean aperture (cm) } & GRD & GRE & GRF \\
\cline { 2 - 8 } & GRA & GRB & GRC & 0.045 & 0.037 & 0.025 \\
\hline 1.0 & 0.020 & 0.031 & 0.032 & 0.047 & 0.047 & 0.038 \\
\hline 2.0 & 0.029 & 0.042 & 0.026 & 0.048 & 0.056 & 0.050 \\
\hline 3.0 & 0.038 & 0.053 & 0.023 & 0.051 & 0.066 & 0.063 \\
\hline 4.0 & 0.047 & 0.063 & 0.021 & 0.053 & 0.075 & 0.074 \\
\hline 5.0 & 0.056 & 0.074 & 0.020 & 0.075 \\
\hline
\end{tabular}


Table 2. Permeability Coefficients of the Five Stages of Shear Displacement for Each Specimen.

\begin{tabular}{c|c|c|c|c|c|c}
\hline \multirow{2}{*}{$\begin{array}{c}\text { Shear disp. } \\
(\mathrm{mm})\end{array}$} & \multicolumn{7}{|c}{ C-permeability coefficient $(\mathrm{cm} / \mathrm{sec})$} \\
\cline { 2 - 7 } & GRA & GRB & GRC & GRD & GRE & GRF \\
\hline 1.0 & $7.46 \mathrm{E}-03$ & $1.67 \mathrm{E}-03$ & $1.68 \mathrm{E}-01$ & $1.43 \mathrm{E}-01$ & $5.05 \mathrm{E}-01$ & $5.31 \mathrm{E}-03$ \\
\hline 2.0 & $2.28 \mathrm{E}-03$ & $2.00 \mathrm{E}-03$ & $4.67 \mathrm{E}-04$ & $4.45 \mathrm{E}-01$ & $2.35 \mathrm{E}-02$ & $5.31 \mathrm{E}-03$ \\
\hline 3.0 & $7.18 \mathrm{E}-03$ & $7.79 \mathrm{E}-03$ & $2.32 \mathrm{E}-04$ & $7.60 \mathrm{E}-02$ & $1.16 \mathrm{E}-01$ & $4.16 \mathrm{E}-04$ \\
\hline 4.0 & $1.79 \mathrm{E}-02$ & $1.79 \mathrm{E}-03$ & $1.91 \mathrm{E}-04$ & $1.48 \mathrm{E}-03$ & $5.09 \mathrm{E}-02$ & $3.22 \mathrm{E}-03$ \\
\hline 5.0 & $1.55 \mathrm{E}-01$ & $1.67 \mathrm{E}-03$ & $1.83 \mathrm{E}-04$ & $5.94 \mathrm{E}-04$ & $6.83 \mathrm{E}-03$ & $1.76 \mathrm{E}-03$ \\
\hline
\end{tabular}
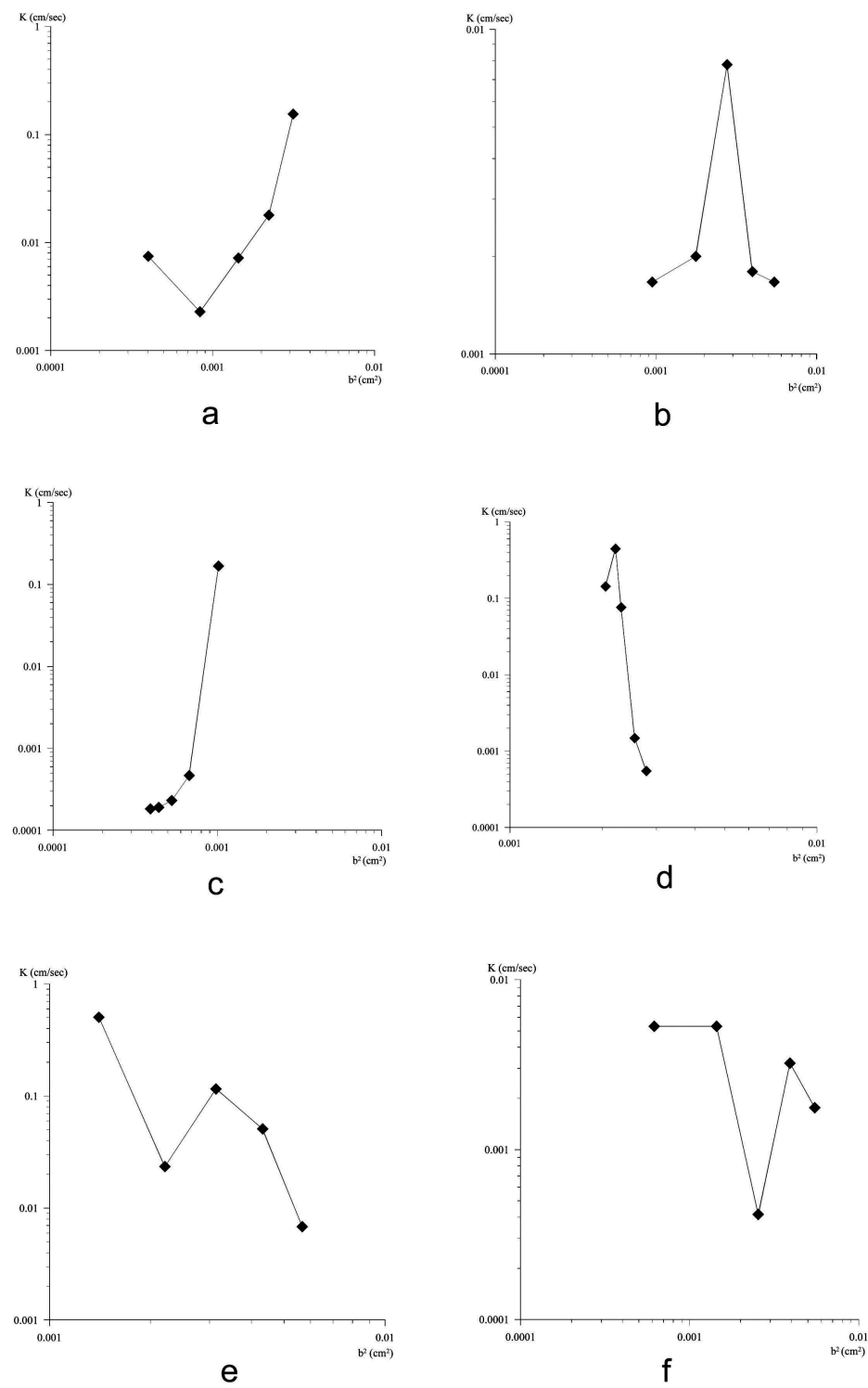

Fig. 7. Relationship between the C-permeability Coefficients and the Aperture Square. (a) GRA, (b) GRB, (c) GRC, (d) GRD, (e) GRE, and (f) GRF 
Case GRD shows a negative relationship between the mean aperture value and the permeability coefficient. During the first stage of the displacement, the GRD case was similar to the parallel plate model. However, this model exhibited an irregular aperture distribution as the displacement increased. At stage 5, the walls were close to being in contact with one another at four points. This geometry did not provide an effective conduit for water flow along the fracture. Consequently, the permeability coefficient was reduced, and a non linear relationship was observed between the mean aperture value and the permeability coefficient.

\section{DISCUSSION}

The distributions of the characteristic velocity, $V_{i}^{k}$, and the characteristic pressure, $p^{k}$, in the fracture models were calculated using the FEM in the procedure to obtain the permeability coefficient $K_{i j}$. In the case of GRA, the result of the permeability calculation indicates that the characteristic velocity, $V_{i}^{k}$, and the characteristic pressure, $p^{k}$, were in agreement with the fracture geometry. Compared with the fracture model of stage 1 for GRA, shown in Figure 6, portions of the wide apertures had lower levels of characteristic pressure than the adjacent parts (Figure 8). The characteristic velocity also exhibited various directions in the model. This variance indicates that there was a turbulent flow of water induced by the rough fracture geometry.

The purpose of this calculation in a unit cell is to understand the distribution of the velocity and pressure characteristic functions on a micro-scale. Only after these calculations is it possible to understand the distributions of velocity and pressure on the macro-scale and the microscale. The trajectory of a fluid flow can be inferred in a large rock mass. Therefore, the flow can be calculated from the micro-scale to the macro-scale in rock fractures.

According to Chae et al. (2003) [43] and Chae (2004) [42], the permeability coefficients that were calculated using the rough fracture models and the parallel plate models are very different. Therefore, it is impossible to compute the accurate permeability coefficients without thoroughly quantifying fracture geometry. Because the fluid flow has different characteristics in a homogeneous porous medium compared with along a rough fracture, it is necessary to analyze the detailed fracture geometry to identify the changes in the permeability characteristics and to obtain accurate permeability coefficients [5,44-46].

To calculate more accurate permeability coefficients, a new numerical method that is different from the empirical equation based on the cubic law is required to analyze the permeability characteristics of a rough fracture. In particular, because the geometric condition of a fracture changes irregularly, the fracture geometry should be considered during the numerical analysis. On the basis of these conditions, the homogenization analysis method is suggested as an appropriate numerical method for the analysis of fracture permeability. Because the HA method considers the fracture geometry on the micro-scale and the macro-scale, it is possible to understand the permeability characteristics, including the local effect and the overall influence of a fracture. Therefore, the HA method can more accurately determine the permeability characteristics for complicated fracture properties than can the previous empirical equations.

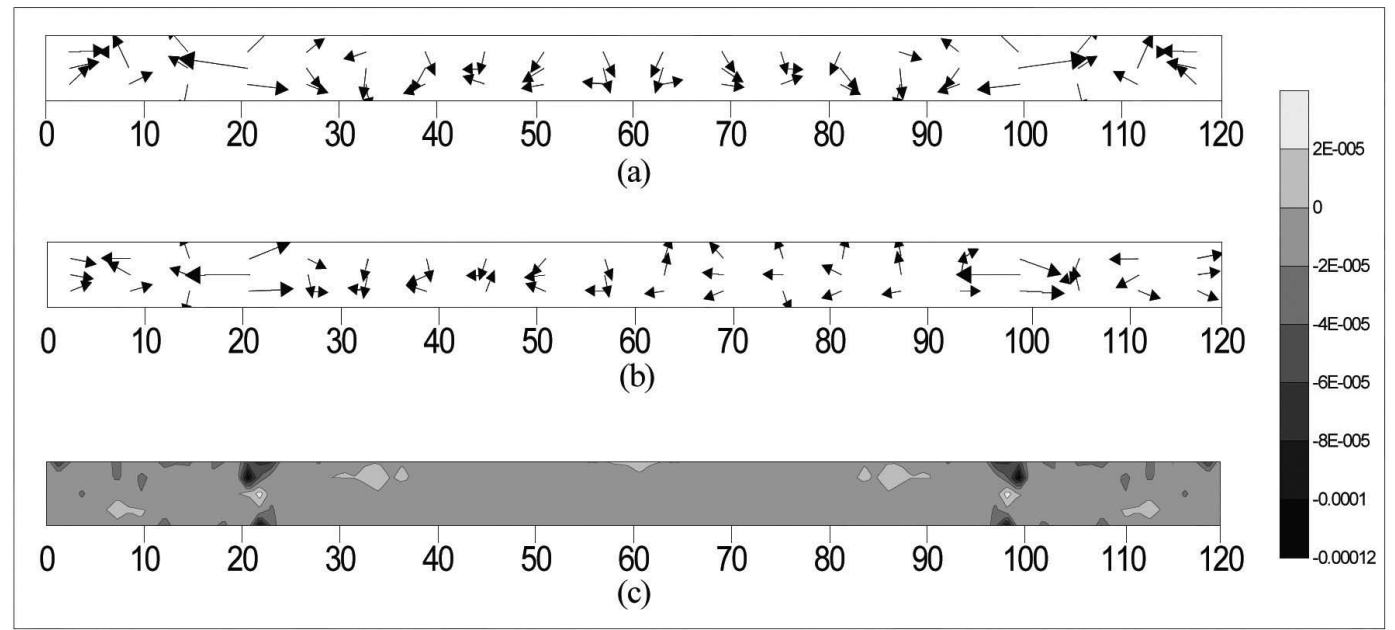

Fig. 8. Distribution of the Characteristic Velocity and the Characteristic Pressure in a Fracture Unit Cell. (a) Characteristic Velocity of $v_{x}^{k}$, (b) Characteristic velocity of $v_{y}^{k}$, and (c) Characteristic pressure $p^{k}$ 


\section{CONCLUSION}

In the present study, the fracture roughness was measured in detail using the CLSM. Using a Fourier spectral analysis, the measured data were analyzed to identify the effective geometrical characteristics. Low pass filtered roughness profiles were reconstructed using the effective frequencies. The reconstructed profiles were applied to 2-D fracture models of permeability using the homogenization analysis (HA).

To characterize the hydraulic conductivity, the permeability along a single fracture was computed using the HA method and the measured fracture geometry data. Taking into consideration the change of the roughness pattern along a fracture, the permeability coefficient was calculated using the rough fracture models. The upper wall was assumed to be displaced by shearing during the five stages. The calculation results revealed various changes in the permeability that depended on the roughness patterns and the aperture values. The irregular distribution of the roughness and the aperture along a fracture may introduce a negative proportional relationship between the aperture and the permeability coefficient, even though the mean aperture becomes larger. This relationship clearly demonstrates that the permeability characteristics are very sensitive to the geometry of the roughness and the aperture. The characteristic velocity and characteristic pressure functions of the HA were also calculated in order to understand the micro-/macro-properties of the fracture permeability. The results of the HA-simulation were in agreement with the fracture geometry.

On the basis of the above results, the homogenization analysis method is suggested as an appropriate numerical method for the analysis of fracture permeability. Because the HA method considers the fracture geometry on the micro- and macro-scale, this method can be used to understand the permeability characteristics, such as the local effect and the overall influence, of a fracture. Therefore, the HA method can provide more accurate results for the permeability characteristics of complicated fracture properties than the previous empirical equations.

\section{ACKNOWLEDGEMENTS}

This work was supported by the Radioactive Waste Management Program of the Korea Institute of Energy Technology Evaluation and Planning (KETEP), in a grant funded by the Korean Government Ministry of Knowledge Economy (201017102002B).

\section{REFERENCES}

[ 1 ] Kranz, R.L., Frankel, A. and Engelder, T., "The permeability of whole and jointed Barre Granite," Eos Trans AGU, vol. 58, pp. 1229 (1979).

[2] Louis, C.A., "A study of groundwater flow in jointed rock and its influence on the stability of rock masses," Rock Mechanics Research Report, vol. 10 (1969).
[ 3 ] Renshaw, C.E., "On the relationship between mechanical and hydraulic apertures in rough-walled fractures," Journal of Geophysical Research, vol. 100, pp. 24629-24636 (1986).

[4] Snow, D.T., "A parallel plate model of fractured permeable media," PhD thesis, University of Califonia, Berkeley, USA (1965).

[5] Tsang, Y.W. and Tsang, C.F., "Channel model of flow through fractured media," Water Resources Research, vol. 23, pp. 467-479 (1987).

[6] Brown, S.R., "Fluid flow through rock joints. The effect of surface roughness," Journal of Geophysical Research, vol. 92, pp. 1337-1347 (1987).

[ 7 ] Cook, A.M., Myer, L.R., Cook, N.G.W. and Doyle, F.M., "The effects of tortuosity on flow through a natural fracture," Proceedings of the 31st US Symposium on Rock Mechanics, Colorado, USA, June 18-20, pp. 371-378 (1990).

[ 8 ] Neuzil, C.E. and Tracy, J.V., "Flow through fractures," Water Resources Research, vol. 17, pp. 191-199 (1981).

[9] Piggott, A.R. and Elsworth, D., "Analytical models for flow through obstructed domains," Journal of Geophysical Research, vol. 97, pp. 2085-2093 (1992).

[10] Pyrak-Nolte, L.J., Cook, N.G.W. and Nolte, D.D., "Fluid percolation through single fractures," Geophysical Research Letters, vol. 15, pp. 1247-1250 (1988).

[11] Thompson, M.E. and Brown, S.R., "The effect of anisotropic surface roughness on flow and transport in fractures," Journal of Geophysical Research, vol. 96, pp. 21923-21932 (1991).

[12] Tsang, Y.W. and Witherspoon, P.A., "The dependence of fracture mechanical and fluid flow properties on fracture roughness and sample size," Journal of Geophysical Research, vol. 88, pp. 2359-2366 (1983).

[13] Witherspoon, P.A., Wang, J.S.Y., Iwai, K. and Gale, J.E., "Validity of cubic law for fluid flow in a deformable rock fracture," Water Resources Research, vol. 16, pp. 10161024 (1980).

[14] Zimmerman, R.W., Chen, D. and Cook, N.G.W., "The effect of contact area on the permeability of fractures," Journal of Hydrology, vol. 139, pp. 79-96 (1992).

[15] Brown, S.R., "Transport of fluid and electric current through a single fracture," Journal of Geophysical Research, vol. 94, pp. 9429-9438 (1989).

[16] Paillet, F., Hess, A.E., Cheng, C.H. and Hardin, E., "Characterization of fracture permeability with high resolution vertical flow measurements during borehole pumping," Ground Water, vol. 25, pp. 28-40 (1987).

[17] Walsh, J.B. and Brace, W.F., "The effect of pressure on porosity and the transport properties of rock," International Journal of Rock Mechanics and Mining Sciences \& Geomechanics Abstract, vol. 18, pp. 429-435 (1984).

[18] Taylor, W.L., Pollard, D.D. and Aydin, A., "Fluid flow in discrete joint sets: Field observations and numerical simulations," Journal of Geophysical Research, vol. 104, pp. 28983-29006 (1999).

[19] Gale, J.E., "The effects of fracture type (induced versus natural) on the stress-fracture closure-fracture permeability relationships," Proceedings of the 23rd US Symposium on Rock Mechanics, Berkeley, California, USA, August 25-27, pp. 290-298 (1982a).

[20] Iwai, K., "Fundamental studies of fluid flow through a single fracture," PhD thesis, University of Califonia, Berkeley, USA (1976). 
[21 ] Olsson, W.A., "The effect of slip on the flow of fluid through a fracture," Geophysical Research Letters, vol. 19, pp. 541-543 (1992).

[22] Raven, K.G., and Gale, J.E., "Water flow in a natural rock fracture as a function of stress and sample size," International Journal of Rock Mechanics and Mining Sciences \& Geomechanics Abstract, vol. 22, pp. 251-261 (1985).

[23] Trimmer, D., Bonner, B., Heard, H.C. and Duba, A., "Effect of pressure and stress on water transport in intact and fractured gabbro and granite," Journal of Geophysical Research, vol. 85, pp. $7059-7071$ (1980).

[24] Zimmerman, R.W. and Bodvarsson, G.S., "Hydraulic conductivity of rock fractures," Transport in Porous Media, vol. 23, pp. 1-30 (1996).

[25] Brown, S.R. and Scholz, S.H., "Broad bandwidth study of the topography of natural rock surfaces," Journal of Geophysical Research, vol. 90, pp. 12575-12582 (1985).

[26] Develi, K., Babadagli, T. and Comlekci, C., "A new computer-controlled surface-scanning device for measurement of fracture surface roughness," Computers and Geosciences, vol. 27, pp. 265-277 (2001).

[27] Durham, W.B. and Bonner, B.P., "PEAK : A new kind of surface microscope," International Journal of Rock Mechanics and Mining Sciences \& Geomechanics Abstract, vol. 30, pp. 699-702 (1993).

[28] International Society for Rock Mechanics Commission on Standardization of Laboratory and Field Tests, International Journal of Rock Mechanics and Mining Sciences \& Geomechanics Abstract, vol. 15, pp. 319-368 (1978).

[29] Keller, K. and Bonner, B.P., "Automatic, digital system for profiling rough surfaces," Rev Sci Instrument, vol. 56, pp. 330-331 (1985).

[30] Kulatilake, P.H.S.W., Shou, G., Huang, T.H. and Morgan, R.M., "New peak shear strength criteria for anisotropic rock joints," International Journal of Rock Mechanics and Mining Sciences \& Geomechanics Abstract, vol. 32, pp. 673-697 (1995).

[31] Lee, Y.H., Carr, J.R., Barr, D.J. and Haas, C.J., “The fractal dimension as a measure of the roughness of rock discontinuity profiles," International Journal of Rock Mechanics and Mining Sciences \& Geomechanics Abstract, vol. 27, pp. 453-464 (1990).

[32] Lespinasse, M. and Sausse, J., "Quantification of fluid flow: hydro-mechanical behaviour of different natural rough fractures," Journal of Geochemical Exploration, vol. 69-70, pp. 483-486 (2000).

[33] Plouraboue, F., Kurowski, P., Boffa, J.-M., Hulin, J.-P. and Roux S., "Experimental study of the transport properties of rough self-affine fractures," Journal of Contaminant Hydrology, vol. 46, pp. 295-318 (2000).
[34] Power, W.L. and Durham, W.B., "Topography of natural and artificial fractures in granitic rocks: implications for studies of rock friction and fluid migration," International Journal of Rock Mechanics and Mining Sciences, vol. 34, pp. 979-989 (1997).

[35] Jesselle, M.W., Cox, S.J.D., Schwarze, P. and Power, W., "The anisotropy of surface roughness measured using a digital photogrammetric technique," Special Pub Geol Soc London, vol. 92, pp. 27-37 (1995).

[36] Krohn, C.E. and Thompson, A.H., "Fractal sandstone pores: automated measurements using scanning-electronmicroscope images," Physical Review, B 33, pp. 63666374 (1986).

[37] Maerz, N.H., Franklin, J.A. and Bennett, C.P., "Joint roughness measurement using shadow profilometry," International Journal of Rock Mechanics and Mining Sciences \& Geomechanics Abstract, vol. 27, pp. 329-343 (1990).

[38] Ichikawa, Y., Kawamura, K., Nakano, M., Kitayama, K. and Kawamura, H., "Unified molecular dynamics and homogenization analysis for bentonite behavior: current results and future possibilities," Engineering Geology, vol. 54, pp. 21-31 (1999).

[39] Sanchez-Palencia, E., Non-homogeneous media and vibration theory, Springer-Verlag (1980).

[40] Chae, B.G., Ichikawa, Y., Jeong, G.C., Seo, Y.S. and Kim, B.C., "Roughness measurement of rock discontinuities using a confocal laser scanning microscope and the Fourier spectral analysis," Engineering Geology, vol. 72, pp. 181-199 (2004).

[41] Ohsaki, Y., Introduction to spectral analysis of seismic motion, Kajima Publication (1981).

[42] Chae, B.G., "Application of the homogenization analysis to calculation of a permeability coefficient," The Journal of Korean Society of Soil and Groundwater Environment, vol. 9, pp. 79-86 (2004).

[43] Chae, B.G., Ichikawa, Y., Jeong, G.C. and Seo, Y.S., "Aperture of Granite Fracture and Effects for Fluid Flow," Materials Science Research International, vol. 9, pp. 270277 (2003).

[44] Gale, J.E., "Assessing the permeability characteristics of fractured rock," GSA. Special Paper, vol. 189, pp. 163-181 (1982b).

[45] Tsang, Y.W. and Witherspoon, P.A., "Hydromechanical behavior of a deformable rock fracture subject to normal stress," Journal of Geophysical Research, vol. 86, pp. 9287-9298 (1981).

[46] Tsang, Y.W., Tsang, C.F., Neretnieks, I. and Moreno, L., "Flow and tracer transport in fractured media: A variable aperture channel model and its properties," Water Resources Research, vol. 24, pp. 2049-2060 (1988). 